\title{
Dynamic Error Correction of Methane Sensor
}

\author{
Beata Krupanek and Ryszard Bogacz
}

\begin{abstract}
Coal mine methane is a term given to the methane gas produced or emitted in association with coal mining activities either from the coal seam itself or from other gassy formations underground. The primary reason for measuring methane is to improve the safety of the mines. In recent years, there have been many fatalities in underground coal mine explosions in which methane was a contributing factor. The rapid detection of methane is very important from the point of view of safety of mine workers. This paper presents a concept of fast methane detection by reconstituting its concentration in dynamic states.
\end{abstract}

Keywords - dynamic error correction, methane sensor, pellistor sensor, safety in mines

\section{INTRODUCTION}

$\mathbf{M}$ ETHANE is a dangerous gas which occurs mainly in coal mines. The concentration of methane in some zones may pass through a range between 5\% and 15\%, known as the explosive range. In this range, methane can be ignited easily with the presence of an ignition source to create a violent methane explosion that may propagate in the presence of combustible coal dust [20]. Coal mine methane has always been considered as a danger for underground coal mining as it can create a serious threat to mining safety and productivity due to its explosion risk [17].

The amount of methane generated at a specific operation depends on the productivity of the coal mine, the gassiness of the coal seam and any underlying and overlying formations, operational variables, and geological conditions. One of the most important duties of ventilation in underground coal mines is to keep methane levels well below the explosive limit by diluting methane emissions that occur during mining. From these reasons the methane concentration should be controlled by continuous measurements [9, 12].

Methane sensors use different detection technologies according to the three gas categories and these are pellistors, electrochemical, and infrared devices [2]. Typically pellistors are used for detecting methane and any flammable gas [4, 19]. One of the disadvantages of pellistor sensor is a long response time $[10,13]$. This defect can be significantly reduced by using a dynamic correction method $[14,15,16]$. Such a method was shown in [1]. Article shows a way for use the correction method for pellistor sensors in coal mines for reducing the time to detect methane.

B. Krupanek is from Silesian University of Technology, Faculty of Electrical Engineering, Institute of Measurement Science, Electronics and Control, Akademicka 10, 44-100 Gliwice, Poland (e-mail: beata.krupanek@polsl.pl).

R. Bogacz is from Silesian University of Technology, Faculty of Electrical Engineering, Institute of Measurement Science, Electronics and Control, Akademicka 10, 44-100 Gliwice, Poland (e-mail: ryszard.bogacz@polsl.pl).

\section{PRINCIPLE OF OPERATION OF PELLISTOR SENSOR}

Pellistors are gas sensors that detect combustible gases and vapours in air (or atmospheres containing oxygen), in concentrations approaching the explosive range. In this case, they are operating in a catalytic mode where the target gas is burnt and the heat liberated is measured by the sensor. The working principle of catalytic sensors is based on flammable gas oxidation: when a combustible gas comes in contact with the catalyst surface it is oxidised. The reaction releases heat, which causes the resistance of the wire to change.

A catalytic palletised resistor (or "Pellistor") consists of a very fine coil of platinum wire, embedded within a ceramic pellet (fig.1). On the surface of the pellet is a layer of a high surface area noble metal, which, when hot, acts as a catalyst to promote exothermic oxidation of flammable gases. This active bead is known as the Detector bead. In order to compensate for environmental factors such as temperature and pressure, the active bead is matched with an inactive reference or Compensator bead. This pair of beads is generally run in a simple Wheatstone bridge circuit. The signal produced is proportional to the gas concentration up to the lower explosive limit, or LEL [6]. The sensor will detect all combustible gases and vapours, although the response to higher hydrocarbons may not be high [11].
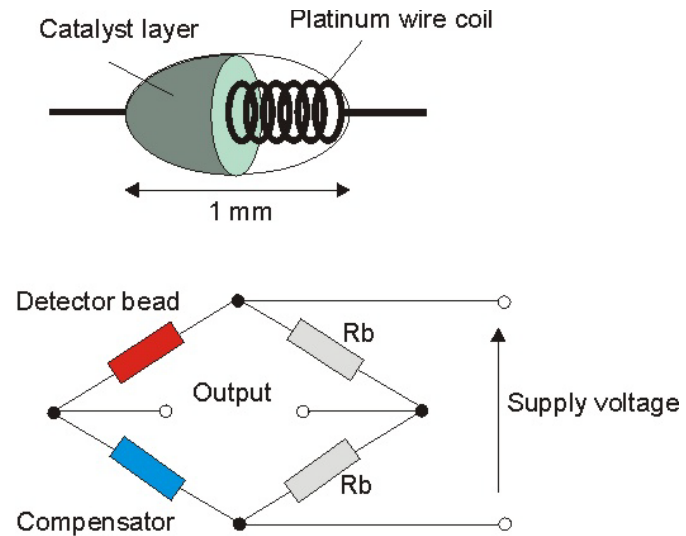

Fig. 1. Pellistor gas sensor and a build of measuring head (Wheatstone bridge)

The thermal conductivity sensor comprises two beads, one of which is exposed to the target gas (the Detector) and the other sealed inside a chamber containing air (the Compensator). Both beads are heated as before and run in the same type of circuit as the catalytic sensor. When the detector bead is exposed to a gas whose thermal conductivity is significantly different to that of air, the rate of heat loss from the bead will change, as will its resistance. This change measured is compared with the compensator bead [4].

The catalytic sensor is excellent for the detection of a wide range of combustible gases within the Lower Explosive Limit. The response time depends on the gas being detected: the greatest is the gas weight or the molecular dimension the 
longer will be the response time. A T90 time response to methane detection is usually provided in 5 - 10 seconds; a slight increase occurs when the sensor is protected by a sintered filter $[11,12]$.

Because of catalytic combustion the pellistor sensor must be placed behind a flameproof enclosure [5]. It normally contains a sintered metal flame arrester that also acts as a primary diffusion barrier through which the gas must pass before reaching the sensing element [3, 7]. Sintered metal materials are a popular choice because these highly engineered materials consist of a uniform, interconnected porosity that can be fabricated to allow precise gas flow control while providing a non-shedding, mechanically sound, media to quench the flame front. Sintered metal provides cost effective solutions for high volume devices, especially for applications that require the prevention of a flame front in the event that an intense explosion occurs. Porous sintered metal media can also be formed into net shapes and easily joined to hardware to create unique assemblies [6].

Having passed through this initial barrier, there is a second (less critical) barrier at the entrance to the sensor can before the gas reaches the vicinity of the sensor beads. Once near the catalytic detector bead, there is a third barrier that is formed by diffusion of combustion products away from the detector that produces a depletion layer around the surface of the detector. This depletion layer changes the rate of diffusion of fuel to the catalyst surface. All of this factors cause the much longer time to receive the stable value of a concentration of methane in the case of its rapid growth [18]

\section{DYNAMIC CORRECTION OF METHANE CONCENTRATION}

Measurement errors can be divided into two types: static and dynamic [8]. Static errors are the ones that cause registration errors even when the user's viewpoint and the objects in the environment remain completely still. Dynamic errors are the ones that have no effect until either the viewpoint or the objects begin moving. Correcting for dynamic errors is important because they are usually the largest contributors to measurements error. Dynamic errors are primarily caused by system delays. In this case dynamic error is caused by delay introducing by flameproof arrester. This delay can be variable because the shield is placed in dusty and polluted places in mine. Changes of time constant may be large what illustrates the figure 2 .

Determination of a dynamic correction algorithm of a real measuring sensor requires knowledge of its dynamics (time constant) in the form of differential equations and of the dynamics the entire measurement system. Therefore, correction algorithms must always be determined for specific measuring transducers and measurement systems, and the resultant equations must only be applied to the particular sensors. Prediction or correction is an important technique to compensate for system delays. Nowadays says about programming correction because of evolution of microcontrollers witch realizes the mathematical calculations almost in real time.

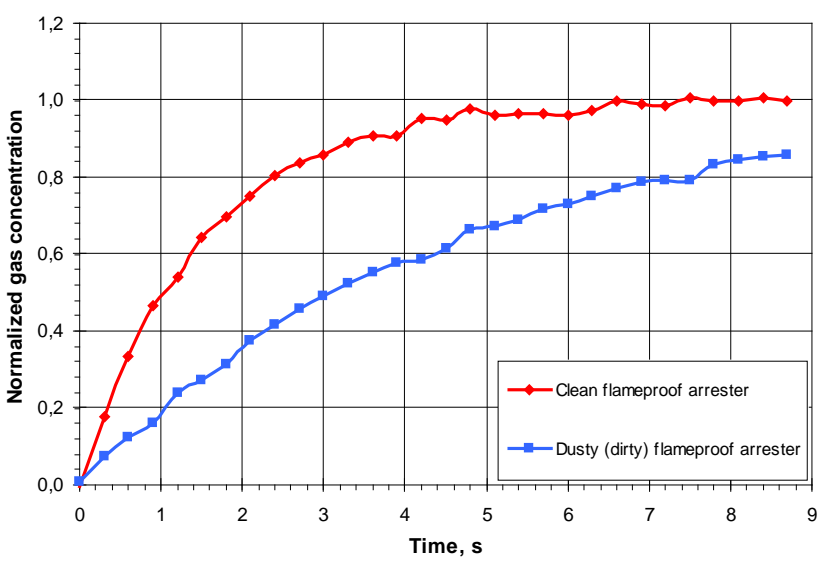

Fig. 2. Changes of pellistor sensor time constant

Dynamic properties of methane sensor can be modeled by a linear differential equation of $n$-th order

$$
\frac{1}{a_{0}}\left[y^{(n)}+a_{n-1} y^{(n-1)}+\cdots+a_{1} \dot{y}\right]+y=x,
$$

where: $a_{n-1}, \ldots, a_{0}$ are constant coefficients, $x$ is the input quantity (methane concentration), $y$ is the sensor output quantity. This equation can be written as:

$$
y=x+\delta_{d y n},
$$

where

$$
\delta_{d y n}=-\frac{1}{a_{0}}\left[y^{(n)}+a_{n-1} y^{(n-1)}+\cdots+a_{1} \dot{y}\right]
$$

is an expression that describes the dynamic processing error. When changes of $y$ quantity occurs respectively slow, the parts of expression (3) containing derivatives take values close to zero and equation (2) takes the form of the equation of ideal processing. According to formula (2) sensor output can be represented as the sum of the input quantity and dynamic error specified by expression (3). This relationship allows us to determine the dynamic error at the output of the transducer to any changes of known input quantity.

In the case when transducer can be described by first order equation, it takes the form

$$
\dot{y}+a_{0} y=b_{0} x,
$$

where $a_{0}=b_{0}$ when the equation (4) is ideal statically (static sensitivity is equal to 1$)$. In such a situation

$$
T \dot{y}+y=x,
$$

where $T=\frac{1}{a_{0}}$ is a sensor time constant.

Dynamic correction is usually realized by microcontroller due to its numerical complexity. We assume that the measured value $x$ is converted to quantity $y$ and the model of this processing can be described by function:

$$
y=f(x) \text {. }
$$


Dynamic correction is based on solving the inverse function to (6) witch is taken from measurement of the $y$ quantity according to the equation

$$
\hat{x}=f^{-1}(y) \text {. }
$$

Combining equations (6) and (7) we obtain

$$
\hat{x}=f^{-1}(y)=f^{-1}[f(x)]=x,
$$

which means that the output values $\hat{x}$ are equal to the values of the measured quantity. It could be said that the measuring circuit performs tasks of ideal transducer. Assuming that the above measures are performed by ADC then the equation (8) takes the form:

$$
\hat{x}\left(t_{\mathrm{k}}\right)=x\left(t_{\mathrm{k}}\right)
$$

which means that the instantaneous values $\hat{x}\left(t_{\mathrm{k}}\right)$ of the transducer output repeat the instantaneous values of the measured quantity $x\left(t_{\mathrm{k}}\right)$ at times $t_{\mathrm{k}}$, where $k$ is the number of sampling times, $k=0,1, . ., n$.

In general case the showed algorithm has a recurrent solution witch was described in [1]. First order model is a special case, for which there is no form of recursive algorithm correction and the algorithm has simply form

$$
\hat{x}(k)=A_{k+1} \tilde{y}(k+1)+A_{k} \tilde{y}(k) .
$$

The values of the coefficients from equation (10) can be determined as follows:

$$
\begin{aligned}
& A_{k+1}=\frac{1}{1-\varphi}, \\
& A_{k}=\frac{-\varphi}{1-\varphi},
\end{aligned}
$$

where $\varphi=\mathrm{e}^{-\frac{T_{\mathrm{d}}}{T}}, T_{\mathrm{d}}$ is a sampling period and $T$ is a time constant of first order differential equation.

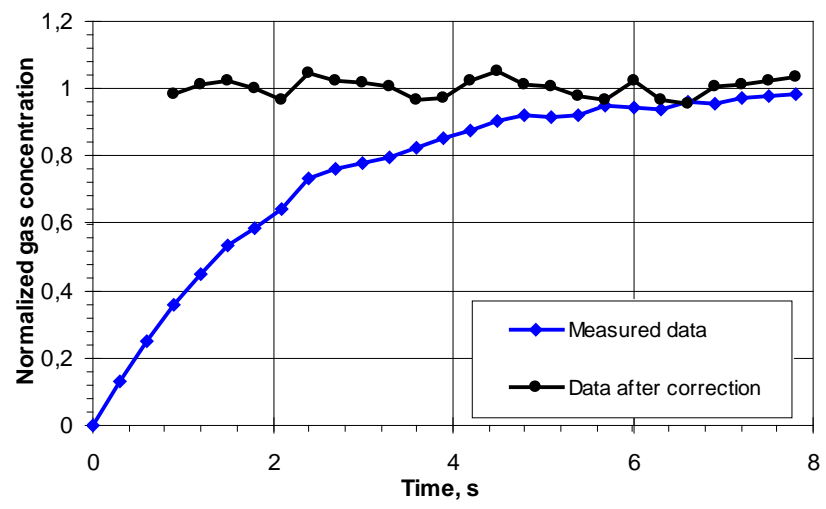

Fig. 3. Results of dynamic correction for methane concentration

Figure 3 shows a experimental results of methane dynamic correction for the sudden change in the concentration of methane. It can be seen than the algorithm of correction works properly with a delay equal to two sampling periods. This accelerate the measurement almost several times.

\section{CONCLUSIONS}

Method described in this paper lead to the conclusion that it is possible the current dynamic error correction of penetration of methane by flameproof arrester, based on the instantaneous values of methane concentration inside the enclosure measured using pellistor sensor. Correction is performed on the basis of dynamic correction of the discrete changes of the concentration outside the enclosure, and the result is a significant decrease in time to obtain a result. This method can significantly helps to improvement the work safety of miners.

\section{REFERENCES}

[1] R. Bogacz, B. Krupanek, "Selected methods for acceleration the measurements of gas concentration in transient states”, Pomiary Automatyka Kontrola, 1/2014, pp.26-30 (in polish).

[2] J. Chou, "Hazardous Gas Monitors", A Practical Guide to Selection, Operation and Applications, McGraw-Hill Book Company, New York, 2000.

[3] J. Crank, “The mathematics of diffusion”, Oxford University Press, New York, 1993.

[4] J. Fraden, "Handbook of modern sensors: physics, designs, and applications”, Jacob Fraden-3rd ed., Springer, 2004.

[5] S. Grossel, "Deflagration and Detonation Arresters", Center for Chemical Process Safety/AIChE, 2002.

[6] R. E. Henderson, "Understanding Combustible Sensor Performance", International Fire Protection 2006 v.27 pp.55-59.

[7] K. H. Clifford, S. W. Webb, "Gas transport in porous media”, Springer, 2006.

[8] J. Jakubiec, “A new conception of measurement uncertainty calculation”, Acta Physica Polonica A, vol.124, 2013, pp. 436-444.

[9] F. N. Kissell, “Handbook for Methane Control in Mining”, Department of Health and Human Services, Information Circular, 2006.

[10] S. D. Kolev, M. Adam, I. Barsony, A. van den Berg, C. Cobianu, "Mathematical modelling of porous silicon based pellistor-type catalytic flammable gas sensors”, Microelectronics Journal 29/1998, pp.235-239.

[11] M. Krawczyk, J. Namiesnik, "Application of a catalytic combustion sensor (pellistor) for the monitoring of the explosiveness of a hydrogenair mixture in the upper explosive limit range", Journal of Automated Methods \& Management in Chemistry, vol.25, no.5, pp.115-122.

[12] A. Kumar et all, "Application of gas monitoring sensors in underground coal mines and hazardous areas”, International Journal of Computer Technology and Electronics Engineering, vol.3, Iss.3, 2013, pp.9-23.

[13] G. McRobbie, F. Clark, C. Tandy, "The Performance of a Flammable Gas Sensing Pellistor Bead With Respect to the Material Properties of the Support Arms”, International ANSYS Conference, 2002.

[14] J. Nabielec, J. Nalepa, "The 'Blind' Method of Dynamic Error Correction for Second Order System”, Proceedings of XVII IMEKO World Congress, 2003, pp.841-846.

[15] J. Nabielec, J. Nalepa, “A Simulation Investigation of Differential Algorithm for the "Blind Correction" of Dynamic Error in Measuring Channels with Periodic Nonstationarity”, Proc. of XVIII IMEKO World Congress, 2006.

[16] J. Nalepa, “Correction of Dynamic Error by the „Blind” Method. A Differential Algorithm Simulation Study”, Proc. of 10th IMEKO TC7 Int. Symp. on Advances of Measurement Science, 2004, pp.109-114.

[17] K. Noack, "Control of gas emissions in underground coal mines", International Journal of Coal Geology 35, 1998, pp.57-82.

[18] G. Rose, I. Zdanevitch, "A New Method Using a Catalytic Sensor for the Identification and Concentration Measurement of Combustible Gases”, Sensors and Actuators B, vol. 24-25, 1995, p.426-428.

[19] G. Rose, "Microsensors for Methane and Other Combustible Gases", Eurosensors XI, The 11-th European Conference on Solid State Transducers, Warsaw, Poland, SEPT 21-24, 1997, pp.123-126.

[20] C. D. Taylor, J. E. Chilton, G. V. R. Goodman, "Guidelines for the Control and Monitoring of Methane Gas on Continuous Mining Operations”, Department of Health and Human Services, Information Circular, 2010. 